\title{
Poynting's theorem and luminal total energy transport in passive dielectric media
}

\author{
Scott Glasgow \\ Glasgow@mathematics.byu.edu \\ Michael Ware \\ ware@byu.edu \\ Justin Peatross \\ peat@byu.edu
}

Follow this and additional works at: https://scholarsarchive.byu.edu/facpub

Part of the Astrophysics and Astronomy Commons, and the Physics Commons

\section{Original Publication Citation}

Physical Review E, vol 64, 4661, pp 1-14.

\section{BYU ScholarsArchive Citation}

Glasgow, Scott; Ware, Michael; and Peatross, Justin, "Poynting's theorem and luminal total energy transport in passive dielectric media" (2001). Faculty Publications. 563.

https://scholarsarchive.byu.edu/facpub/563

This Peer-Reviewed Article is brought to you for free and open access by BYU ScholarsArchive. It has been accepted for inclusion in Faculty Publications by an authorized administrator of BYU ScholarsArchive. For more information, please contact ellen_amatangelo@byu.edu. 


\title{
Poynting's theorem and luminal total energy transport in passive dielectric media
}

\author{
S. Glasgow, ${ }^{1}$ M. Ware, ${ }^{2}$ and J. Peatross ${ }^{2}$ \\ ${ }^{1}$ Department of Mathematics, Brigham Young University, Provo, Utah 84601 \\ ${ }^{2}$ Department of Physics, Brigham Young University, Provo, Utah 84601 \\ (Received 26 June 2000; revised manuscript received 17 May 2001; published 25 September 2001)
}

\begin{abstract}
Without approximation the energy density in Poynting's theorem for the generally dispersive and passive dielectric medium is demonstrated to be a system total dynamical energy density. Thus the density in Poynting's theorem is a conserved form that by virtue of its positive definiteness prescribes important qualitative and quantitative features of the medium-field dynamics by rendering the system dynamically closed. This fully three-dimensional result, applicable to anisotropic and inhomogeneous media, is model independent, relying solely on the complex-analytic consequences of causality and passivity. As direct applications of this result, we show (1) that a causal medium responds to a virtual, "instantaneous" field spectrum, (2) that a causal, passive medium supports only a luminal front velocity, (3) that the spatial "center-of-mass" motion of the total dynamical energy is also always luminal and (4) that contrary to (3) the spatial center-of-mass speed of subsets of the total dynamical energy can be arbitrarily large. Thus we show that in passive media superluminal estimations of energy transport velocity for spatially extended pulses is inextricably associated with incomplete energy accounting.
\end{abstract}

DOI: 10.1103/PhysRevE.64.046610

PACS number(s): 42.25.Bs

\section{INTRODUCTION}

Recently several groups have published the outcomes of experiments in which superluminal electromagnetic pulse propagation has been observed in various senses. These overages have varied from the moderate [1], to the extreme [2]. In most (but not all) of these recent works the authors have freely expressed the conservative sentiment that nothing particularly disturbing has occurred with respect to relativity. Indeed it is well known that all of the the predictions of the current classes of superluminal phenomenology have been inspired by classical theory, which is heavily circumscribed by the limitations of relativity. One of the purposes of the theoretical work presented here is to point out ways in which these conservative sentiments can be made precise.

To accomplish this, we address and clarify the central issue of energy transport in dissipative/dispersive dielectrics. (Here we limit to the passive case and address the active case elsewhere [3].) We make these clarifications by introducing a new theorem and an immediate corollary that address the phenomena of global energy flow in causal media. It is only in this global sense that the various authors have ventured to predict and, recently, to verify superluminal electromagnetic pulse propagation, the local sense having been authoritatively proscribed by the theorems of Sommerfeld and Brillouin [4] almost 85 years ago. (The global theory presented here also contains the main implication of the local Sommerfeld-Brillouin theory as an important corollary.)

In order to produce a notion of global energy transport that is unambiguous, we employ the method of moments or expectations (more often seen and used in quantum mechanics and kinetic theory than in electromagnetic theory). These techniques allow one to pass beyond the (often severe) analytic limitations of the local analyses usually employed in this area of research. For example, a commonly employed local tool is the Taylor series. Importantly, many of the objects to which this local tool is applied when analyzing the "superluminal" nature of energy transport in dielectrics do not have series representations that converge in large enough intervals to capture the cause of the anomalous behavior. This is because these effects are associated with mediumfield resonances that are given mathematically by singularities in the relevant constitutive relations. Thus, in order to establish an unambiguous notion of the global properties of energy transport for finite energy medium-field excitations, we introduce the moments of various components of the total energy (analogous to a center of mass). Energy naturally lends itself to this method since expectations are most instructive when the analog of a probability distribution (i.e., a positive definite form) is used. With regard to superluminal phenomena the evolutions of these various moments are not only enlightening and subject to concrete analysis, but also give the relevant and unambiguous generalization of group velocity for arbitrarily complicated pulses [5].

The main results of this paper are given in a theorem and a corollary. The first is given by Eqs. (48)-(50), and the second by Eqs. (88) through (92). Most of this paper is devoted to their development, with only a limited amount of space given to their application. In another publication [3] we show how the theorem can be used to precisely (i.e., quantitatively) explain both the Garrett and McCumber [6] and Chiao [7] effects (as demonstrated through experiment by Chu and Wong [8] and Wang et al. [2], respectively). We also discuss elsewhere [9] how the traditional, local concept of energy transport velocity and the global concept of the velocity of the energy's spatial "center-of-mass" both prescribe upper bounds on the signal velocity.

This paper is organized as follows: in Sec. II we develop Poynting's theorem for a passive dielectric. In Sec. II A we present Maxwell's equations and the assumptions that apply most generally to a passive linear dielectric. In Sec. II B we then show how this structure produces a positive definite form for the total dynamical system energy density. Section II C discusses this form and shows how it implies luminal 
front speed without the usual recourse to path integrals, as well as pointing out the crucial distinctions between the dynamic total energy density and the quantity $\frac{1}{2} \mathbf{E} \cdot \mathbf{D}+\frac{1}{2} \mathbf{B} \cdot \mathbf{H}$, which is sometimes referred to [10]. In Sec. III we present the simple corollary to the theorem of Sec. II B that augments the local Sommerfeld-Brillouin theorems by showing that total energy transport is also globally luminal. Finally, in Sec. IV, and in contrast to the unsurprising results of Sec. III, we show that a certain subset of the total energy can have superluminal global transport properties.

\section{POYNTING'S THEOREM AND CONSERVATION OF TOTAL DYNAMICAL ENERGY}

\section{A. Assumptions}

We start with Maxwell's equations for the four real macroscopic fields. These fields are the electric field $\mathbf{E}(\mathbf{x}, t)$, the electric displacement $\mathbf{D}(\mathbf{x}, t)$, the magnetic induction $\mathbf{B}(\mathbf{x}, t)$, and the magnetic field $\mathbf{H}(\mathbf{x}, t)$. $\mathbf{x}$ and $t$ denote, respectively, the spatial and temporal coordinates. We currently exclude the possibility of macroscopic currents so that we are dealing with a true dielectric. The dynamical equations are then (in the Heaviside-Lorentz system of units)

$$
\begin{gathered}
\frac{\partial}{\partial t} \mathbf{D}(t)-c \boldsymbol{\nabla} \times \mathbf{H}(t)=\mathbf{0}, \\
\frac{\partial}{\partial t} \mathbf{B}(t)+c \boldsymbol{\nabla} \times \mathbf{E}(t)=\mathbf{0} .
\end{gathered}
$$

Here and in much of the following we explicitly denote only the time coordinate since we assume only temporally nonlocal constitutive relations - i.e., we assume temporal but not spatial dispersion. We assume these relations are, nevertheless, local in the frequency domain (stationary in time) and also linear:

$$
\begin{aligned}
& \mathbf{D}(\omega)=\hat{\boldsymbol{\epsilon}}(\omega) \mathbf{E}(\omega), \\
& \mathbf{B}(\omega)=\hat{\mu}(\omega) \mathbf{H}(\omega) .
\end{aligned}
$$

$\hat{\epsilon}$ and $\hat{\mu}$ are, respectively, the (electric) permittivity and (magnetic) permeability tensors. Since we currently exclude nonlinear effects, $\hat{\epsilon}$ and $\hat{\mu}$ are tensors of rank 2 , and since we can think of the fields as three-component column vectors, we can interpret these tensors as $3 \times 3$ matrices. The right hand sides of Eqs. (3) and (4) are then interpreted in the sense of matrix multiplication.

Note that the permittivity and permeability tensors can also depend locally on the space coordinate $\mathbf{x}$,

$$
\begin{gathered}
\hat{\epsilon}=\hat{\epsilon}(\mathbf{x}, \omega), \\
\hat{\mu}=\hat{\mu}(\mathbf{x}, \omega) .
\end{gathered}
$$

We will suppress this dependence for the time being as it does not enter the calculations immediately, but we emphasize that this spatial dependence is important in the end to achieve finite and, hence, physical total energy.
With both the permittivity and permeability tensors nontrivial (i.e., not proportional to the identity) and depending (locally) on the spatial coordinate (as well as nonlocally on time), we are prepared to analyze inhomogeneous and anisotropic media with both electric and magnetic effects. The development of the total energy density in the following section can be greatly simplified leaving out anisotropy, but we include the more general derivation since interest has reemerged recently in considering these effects $[11,12]$.

As is obvious in these constitutive relations, we have adopted the common practice of using the same symbols to denote the fields as well as their temporal Fourier transforms, distinguishing the two sets only by explicit reference to either time $t$ or frequency $\omega$ : for $\mathbf{F}(t)$ any one of the original four fields, we define $\mathbf{F}(\omega)$ via

$$
\mathbf{F}(\omega):=\frac{1}{\sqrt{2 \pi}} \int_{-\infty}^{+\infty} d t e^{i \omega t} \mathbf{F}(t),
$$

and then note the inversion formula

$$
\mathbf{F}(t)=\frac{1}{\sqrt{2 \pi}} \int_{-\infty}^{+\infty} d \omega e^{-i \omega t} \mathbf{F}(\omega)
$$

Since the original fields are real, the transforms manifest the symmetry $\mathbf{F}^{*}(\omega)=\mathbf{F}\left(-\omega^{*}\right)$. Via Eqs. (3) and (4), we then see that the permittivity and permeability tensors possess the same symmetry: e.g., $\hat{\epsilon}^{*}(\omega)=\hat{\epsilon}\left(-\omega^{*}\right)$. In the following, we refer to this symmetry as real symmetry.

In addition to assuming the validity of the macroscopic Maxwell's equations, we limit the constitutive relations (3) and (4) to physically reasonable ones via the following three assumptions.

(a) Causality. $\hat{\epsilon}(\omega)-\hat{I}$ and $\hat{\mu}(\omega)-\hat{I}$ are rapidly vanishing and analytic (termwise) in the upper-half complex $\omega$ plane $(\hat{I}$ is the identity tensor). This implies the Kramers-Kronig relations. Among these we will need that, for real $\omega$,

$$
\begin{gathered}
\operatorname{Re}[\hat{\epsilon}(\omega)]=\hat{I}+\frac{1}{\pi} \mathrm{P} \int_{-\infty}^{+\infty} d \omega^{\prime} \frac{\operatorname{Im}\left[\hat{\epsilon}\left(\omega^{\prime}\right)\right]}{\omega^{\prime}-\omega} \\
\operatorname{Re}[\hat{\mu}(\omega)]=\hat{I}+\frac{1}{\pi} \mathrm{P} \int_{-\infty}^{+\infty} d \omega^{\prime} \frac{\operatorname{Im}\left[\hat{\mu}\left(\omega^{\prime}\right)\right]}{\omega^{\prime}-\omega}
\end{gathered}
$$

Here the symbol $\mathrm{P}$ refers to the operation of taking the Cauchy principal value.

(b) Kinetic symmetry. In the absence of a strong, external, static magnetic field, we have from near-equilibrium thermodynamic considerations [13] that

$$
\begin{aligned}
\hat{\epsilon}^{T}(\omega) & =\hat{\boldsymbol{\epsilon}}(\omega), \\
\hat{\mu}^{T}(\omega) & =\hat{\mu}(\omega) .
\end{aligned}
$$

Here and in the following superscript $T$ indicates the transpose. 
(c) Passivity. We assume that the spectra $\rho$ (i.e., the collection of eigenvalues) of the imaginary parts of $\hat{\epsilon}$ and $\hat{\mu}$ are positive for positive frequencies $\omega$ :

$$
\begin{aligned}
& \rho\{\operatorname{Im}[\hat{\boldsymbol{\epsilon}}(\omega)]\}>0, \\
& \rho\{\operatorname{Im}[\hat{\mu}(\omega)]\}>0 .
\end{aligned}
$$

Note that this assumption of passivity combined with the kinetic symmetry assumption (b) shows that the imaginary parts of the spectra of $\hat{\epsilon}$ and $\hat{\mu}$ are also positive for positive frequencies (which property we call dissipation).

$\left(c^{\prime}\right)$ Dissipation.

$$
\begin{gathered}
\operatorname{Im}[\rho\{\hat{\boldsymbol{\epsilon}}(\omega)\}]>0, \\
\operatorname{Im}[\rho\{\hat{\mu}(\omega)\}]>0 .
\end{gathered}
$$

At first $\left(\mathrm{c}^{\prime}\right)$ might seem a more natural definition of passivity. (E.g., in a crystal the eigenvalues of $\hat{\epsilon}$ give the permittivity in the direction prescribed by the corresponding eigenvectors. The imaginary parts of the eigenvalues then describe absorption.) However, we will eventually see that (c) is the more useful assumption from the complex-analytic point of view. At any rate, in the case that these tensors encode the electromagnetic properties of a crystal or an isotropic medium, (c) and $\left(\mathrm{c}^{\prime}\right)$ are equivalent since the eigenvectors of these tensors can be taken to be real (e.g., the directions of the crystal's principle axes). For a discussion of the relationship between what we have called dissipation and what we have called passivity see the Appendix.

Using real symmetry, we see that the imaginary parts of $\hat{\epsilon}$ and $\hat{\mu}$ are odd functions of real frequency $\omega$. Consequently, according to the passivity property (c) [Eqs. (13) and (14)], we have that for all real frequencies

$$
\begin{aligned}
& \rho\{\omega \operatorname{Im}[\hat{\epsilon}(\omega)]\} \geqslant 0, \\
& \rho\{\omega \operatorname{Im}[\hat{\mu}(\omega)]\} \geqslant 0,
\end{aligned}
$$

with equality possibly holding only at $\omega=0$. We use the fact that these two tensors are non-negative in order to factor them and thereby make their spectral properties obvious: there are tensor-valued functions $\hat{\alpha}_{E}(\omega)$ and $\hat{\alpha}_{H}(\omega)$ such that

$$
\begin{aligned}
& \omega \operatorname{Im}[\hat{\boldsymbol{\epsilon}}(\omega)]=\hat{\alpha}_{E}^{\dagger}(\omega) \hat{\alpha}_{E}(\omega), \\
& \omega \operatorname{Im}[\hat{\mu}(\omega)]=\hat{\alpha}_{H}^{\dagger}(\omega) \hat{\alpha}_{H}(\omega)
\end{aligned}
$$

for all real frequencies $\omega$.

\section{B. Derivation of the total dynamical energy density in Poynting's theorem}

Here we derive the version of Poynting's theorem relevant to the general assumptions made in the preceding section. To our knowledge, this is the first time that this general case has been handled correctly. We begin in the usual way and dot the first of our Maxwell's equations (1) into the electric field $\mathbf{E}(t)$, and add to this the result of dotting the second Eq. (2) into the magnetic field $\mathbf{H}(t)$,

$$
\mathbf{E}(t) \cdot \frac{\partial}{\partial t} \mathbf{D}(t)+\mathbf{H}(t) \cdot \frac{\partial}{\partial t} \mathbf{B}(t)+c \boldsymbol{\nabla} \cdot[\mathbf{E}(t) \times \mathbf{H}(t)]=0 .
$$

Here we have used the usual identity from vector calculus, namely that $\mathbf{H}(t) \cdot \boldsymbol{\nabla} \times \mathbf{E}(t)-\mathbf{E}(t) \cdot \boldsymbol{\nabla} \times \mathbf{H}(t)$ $=\boldsymbol{\nabla} \cdot[\mathbf{E}(t) \times \mathbf{H}(t)]$.

The goal of this section is to express the first two terms in Eq. (21) as the time derivative of a positive definite quantity [quadratic in the electric and magnetic fields, $\mathbf{E}(t)$ and $\mathbf{H}(t)$ ] under the assumptions made in the last section. We will identify this quantity as the total dynamical energy density, comprising recoverable and irrecoverable mechanical energies as well as the energy stored solely in the electromagnetic field. To achieve this goal we temporarily introduce the polarization $\mathbf{P}(t)$ and magnetization $\mathbf{M}(t)$. They are defined (in the Heaviside-Lorentz system of units [10]) via

$$
\begin{aligned}
\mathbf{P}(t) & :=\mathbf{D}(t)-\mathbf{E}(t), \\
\mathbf{M}(t) & :=\mathbf{B}(t)-\mathbf{H}(t) .
\end{aligned}
$$

Using these to eliminate $\mathbf{D}(t)$ and $\mathbf{B}(t)$ from Eq. (21), we obtain

$$
\begin{gathered}
\frac{\partial}{\partial t}\left(\frac{1}{2}\|\mathbf{E}(t)\|^{2}+\frac{1}{2}\|\mathbf{H}(t)\|^{2}\right)+\mathbf{E}(t) \cdot \frac{\partial}{\partial t} \mathbf{P}(t)+\mathbf{H}(t) \cdot \frac{\partial}{\partial t} \mathbf{M}(t) \\
+c \boldsymbol{\nabla} \cdot[\mathbf{E}(t) \times \mathbf{H}(t)]=0 .
\end{gathered}
$$

As the first term of this expression is manifestly the time derivative of a positive definite quadratic form in $\mathbf{E}(t)$ and $\mathbf{H}(t)$, we now need only to recognize the second and third terms in Eq. (24) as such. To that end we introduce and define the electric and magnetic susceptibility tensors $\hat{\chi}_{E}(\omega):=\hat{\epsilon}(\omega)-\hat{I}$ and $\hat{\chi}_{H}(\omega):=\hat{\mu}(\omega)-\hat{I}$. The transforms of the polarization and magnetization vectors, $\mathbf{P}(\omega)$ and $\mathbf{M}(\omega)$, can be expressed locally in terms of the transforms of the electric and magnetic fields via

$$
\begin{aligned}
\mathbf{P}(\omega) & =\hat{\chi}_{E}(\omega) \mathbf{E}(\omega), \\
\mathbf{M}(\omega) & =\hat{\chi}_{H}(\omega) \mathbf{H}(\omega) .
\end{aligned}
$$

Note that from their definitions, and from the relevant properties of the permittivity and permeability tensors [properties (a)-(c)], the susceptibility tensors are analytic and rapidly vanishing in the upper half $\omega$ plane, and also possess properties (b) and (c). They also demonstrate real symmetry: $\hat{\chi}_{F}^{*}(\omega)=\hat{\chi}_{F}\left(-\omega^{*}\right)$. (To avoid repetition, here and in the following $F$ will stand for either $E$ or $H$. Also, owing to the symmetry between the two pairs $(\mathbf{P}, \mathbf{E})$ and $(\mathbf{M}, \mathbf{H})$, in the following we abbreviate by only presenting the derivation of the quadratic form associated with the polarization and electric field. In the end we present the results for both pairs.) 
We next use Eq. (25) to eliminate explicit reference to the polarization vector in Eq. (24). To do this, we inverse Fourier transform (25) to obtain

$$
\mathbf{P}(t)=\int_{-\infty}^{+\infty} d \tau \hat{G}_{E}(t-\tau) \mathbf{E}(\tau),
$$

where the convolution kernel $\hat{G}_{E}(t)$ is defined in terms of the susceptibility via

$$
\hat{G}_{E}(t):=\frac{1}{2 \pi} \int_{-\infty}^{+\infty} d \omega e^{-i \omega t} \hat{\chi}_{E}(\omega) .
$$

We need the time derivative of the polarization. Via Eq. (27), we see that this is obtained through the formula

$$
\frac{\partial}{\partial t} \mathbf{P}(t)=\int_{-\infty}^{+\infty} d \tau \frac{\partial}{\partial t} \hat{G}_{E}(t-\tau) \mathbf{E}(\tau)
$$

(Note: The rapid vanishing of the susceptibilities at large frequencies renders the kernels differentiable everywhere but at a single time where they are, fortunately, continuous. Thus the exchange of orders of the operations of integration and differentiation is justified.)

We now use the various properties of the susceptibilities to reduce Eq. (29) to an equivalent expression that can be used to directly demonstrate the conserved energy. The first (and usual) simplification is to note that the integral (28) can be evaluated explicitly for $t<0$. We use Cauchy's integral theorem with contours constructed from great semicircles in the upper-half $\omega$ plane, closed along the real axis. Since the susceptibilities are analytic and rapidly vanish with increasing radius in the region enclosed by these contours, it is readily shown that for $t<0$ the integration over the real interval defining the convolution kernel gives zero:

$$
\hat{G}_{E}(t)=\hat{0} ; \quad t<0 .
$$

( $\hat{0}$ indicates the zero matrix.) The formula expressing the time derivative of the polarization vector in terms of the electric field, Eq. (29), then reduces to integration up to time $\tau=t$ :

$$
\frac{\partial}{\partial t} \mathbf{P}(t)=\int_{-\infty}^{t} d \tau \frac{\partial}{\partial t} \hat{G}_{E}(t-\tau) \mathbf{E}(\tau)
$$

The previous formula involves the convolution kernel $\hat{G}_{E}$, which is constructed from the susceptibility by Eq. (28). In particular, it appears from that construction that both the real and imaginary parts of the susceptibility are important. We now show that the convolution kernel can be constructed entirely from the imaginary part of the susceptibility which, in turn, will allow us to use passivity (c) to deduce certain important properties of this kernel. To that end, we note that in terms of a susceptibility, the Kramers-Kronig relations [causality (a)] can be expressed as

$$
\operatorname{Re}\left[\hat{\chi}_{F}(\omega)\right]=\frac{1}{\pi} \mathrm{P} \int_{-\infty}^{+\infty} d \omega^{\prime} \frac{\operatorname{Im}\left[\hat{\chi}_{F}\left(\omega^{\prime}\right)\right]}{\omega^{\prime}-\omega}
$$

We can use these relationships between the real and imaginary parts of the susceptibilities to show that the inphase and out-of-phase components of the electric and magnetic convolution kernels are not independent. These two components of the convolution kernels are defined in terms of the real and imaginary parts of the susceptibilities via

$$
\begin{gathered}
\hat{G}_{F}^{\text {in }}(t):=\frac{1}{2 \pi} \int_{-\infty}^{+\infty} d \omega e^{-i \omega t} \operatorname{Re}\left[\hat{\chi}_{F}(\omega)\right], \\
\hat{G}_{F}^{\text {out }}(t):=\frac{i}{2 \pi} \int_{-\infty}^{+\infty} d \omega e^{-i \omega t} \operatorname{Im}\left[\hat{\chi}_{F}(\omega)\right] .
\end{gathered}
$$

Note that $\hat{G}_{F}(t)=\hat{G}_{F}^{\text {in }}(t)+\hat{G}_{F}^{\text {out }}(t)$.

We now show that the in- and out-of-phase components of the convolution kernels are identical for positive argument, i.e., $\hat{G}_{F}^{i n}(t)=\hat{G}_{F}^{\text {out }}(t), t>0$. To that end we rewrite Eq. (33) via Eq. (32) and obtain

$$
\hat{G}_{F}^{i n}(t):=\frac{1}{2 \pi} \int_{-\infty}^{+\infty} d \omega e^{-i \omega t} \frac{1}{\pi} \mathrm{P} \int_{-\infty}^{+\infty} d \omega^{\prime} \frac{\operatorname{Im}\left[\hat{\chi}_{F}\left(\omega^{\prime}\right)\right]}{\omega^{\prime}-\omega} .
$$

Exchanging the orders of the integrations ${ }^{1}$ (and simplifying), we obtain

$$
\hat{G}_{F}^{i n}(t)=\frac{1}{2 \pi^{2}} \int_{-\infty}^{+\infty} d \omega^{\prime}\left(\mathrm{P} \int_{-\infty}^{+\infty} d \omega \frac{e^{-i \omega t}}{\omega^{\prime}-\omega}\right) \operatorname{Im}\left[\hat{\chi}_{F}\left(\omega^{\prime}\right)\right] .
$$

The inner integral can be evaluated via Cauchy's theorem by use of a large semicircular contour that extends into the lower-half plane (for $t>0$ ) and that, for example, contains a small semicircular dimple excluding the pole at $\omega=\omega^{\prime}$. Alternatively, one can recognize the integral as a Hilbert transform and consult a table. Either way the result is that

$$
\mathrm{P} \int_{-\infty}^{+\infty} d \omega \frac{e^{-i \omega t}}{\omega^{\prime}-\omega}=i \pi e^{-i \omega^{\prime} t} ; \quad t>0
$$

Using this result in Eq. (36) gives

$\hat{G}_{F}^{\text {in }}(t)=\frac{i}{2 \pi} \int_{-\infty}^{+\infty} d \omega^{\prime} e^{-i \omega^{\prime} t} \operatorname{Im}\left[\hat{\chi}_{F}\left(\omega^{\prime}\right)\right]=: \hat{G}_{F}^{\text {out }}(t) ; \quad t>0$,

according to definition (34).

Our formula allowing us to eliminate the polarization (31) can now be expressed as

\footnotetext{
${ }^{1} \mathrm{~A}$ rigorous exchange can be made by writing the Cauchy principal value operation as a limit and by restricting the fields to certain physically reasonable function spaces. Similar statements apply to much of what follows.
} 


$$
\frac{\partial}{\partial t} \mathbf{P}(t)=2 \int_{-\infty}^{t} d \tau \frac{\partial}{\partial t} \hat{G}_{E}^{\text {out }}(t-\tau) \mathbf{E}(\tau) .
$$

The advantage of this expression over Eq. (31) is that the auxiliary field is now related to the electric field only through the imaginary part of the susceptibility, about which we have the restrictions of passivity (c). (Recall that we have no direct restriction on the real part of this tensor.)

We are trying to re-express the term $\mathbf{E}(t) \cdot(\partial / \partial t) \mathbf{P}(t)$ in (24) so as to recognize it as the derivative of a positive definite quadratic form in the electric field $\mathbf{E}(t)$. For uniformity of notation between dot products and matrix/tensor products, we will denote this scalar product by juxtaposition of adjoints,

$$
\mathbf{E}(t) \cdot \frac{\partial}{\partial t} \mathbf{P}(t)=\mathbf{E}^{\dagger}(t) \frac{\partial}{\partial t} \mathbf{P}(t)=\left[\frac{\partial}{\partial t} \mathbf{P}(t)\right]^{\dagger} \mathbf{E}(t)
$$

In passing from the second to the third expression we have used that the fields are real.

Using the third form of the expression in Eq. (40) and Eq. (39) to eliminate the auxiliary field $\mathbf{P}$, as well as definition (34) to eliminate the out-of-phase component of the convolution kernel, we find that the dot product can be expressed in terms of only the electric field and the imaginary part of the susceptibility. The formula is

$$
\begin{aligned}
{\left[\frac{\partial}{\partial t} \mathbf{P}(t)\right]^{\dagger} \mathbf{E}(t)=} & \frac{1}{\pi}\left[\int_{-\infty}^{t} d \tau \int_{-\infty}^{+\infty} d \omega\right. \\
& \left.\times e^{-i \omega(t-\tau)} \omega \operatorname{Im}\left[\hat{\chi}_{E}(\omega)\right] \mathbf{E}(\tau)\right]^{\dagger} \mathbf{E}(t)
\end{aligned}
$$

We now remember that, from passivity (c) and real symmetry, $\omega \operatorname{Im}\left[\hat{\chi}_{E}(\omega)\right]$ is a non-negative tensor for all real frequencies [Eq. (19)] and so can be factored,

$$
\begin{aligned}
{\left[\frac{\partial}{\partial t} \mathbf{P}(t)\right]^{\dagger} \mathbf{E}(t)=} & \frac{1}{\pi}\left[\int_{-\infty}^{t} d \tau \int_{-\infty}^{+\infty} d \omega\right. \\
& \left.\times e^{-i \omega(t-\tau)} \hat{\alpha}_{E}^{\dagger}(\omega) \hat{\alpha}_{E}(\omega) \mathbf{E}(\tau)\right]^{\dagger} \mathbf{E}(t)
\end{aligned}
$$

Interchanging the orders of integration and rearranging terms in a more symmetric fashion, we get the suggestive form

$$
\begin{aligned}
{\left[\frac{\partial}{\partial t} \mathbf{P}(t)\right]^{\dagger} \mathbf{E}(t)=} & \frac{1}{\pi} \int_{-\infty}^{+\infty} d \omega\left[\int_{-\infty}^{t} d \tau e^{i \omega \tau} \hat{\alpha}_{E}(\omega) \mathbf{E}(\tau)\right]^{\dagger} \\
& \times e^{i \omega t} \hat{\alpha}_{E}(\omega) \mathbf{E}(t)
\end{aligned}
$$

which is immediately recognized as a sum of the Hermitian products of various vectors with their derivatives:

$$
\begin{aligned}
{\left[\frac{\partial}{\partial t} \mathbf{P}(t)\right]^{\dagger} \mathbf{E}(t)=} & \frac{1}{\pi} \int_{-\infty}^{+\infty} d \omega\left[\hat{\alpha}_{E}(\omega) \int_{-\infty}^{t} d \tau e^{i \omega \tau} \mathbf{E}(\tau)\right]^{\dagger} \\
& \times \frac{\partial}{\partial t}\left[\hat{\alpha}_{E}(\omega) \int_{-\infty}^{t} d \tau e^{i \omega \tau} \mathbf{E}(\tau)\right]
\end{aligned}
$$

This expression would be an obvious perfect derivative if the vectors that are multiplied were not complex conjugates. However, while the individual terms in the frequency integrand are complex, the integration clearly gives a real result. Thus the integrand can be re-expressed in terms of only its real part. We write this as

$$
\begin{aligned}
{\left[\frac{\partial}{\partial t} \mathbf{P}(t)\right]^{\dagger} \mathbf{E}(t)=} & \frac{1}{2 \pi} \int_{-\infty}^{+\infty} d \omega\left\{\left[\hat{\alpha}_{E}(\omega) \int_{-\infty}^{t} d \tau e^{i \omega \tau} \mathbf{E}(\tau)\right]^{\dagger}\right. \\
& \left.\times \frac{\partial}{\partial t}\left[\hat{\alpha}_{E}(\omega) \int_{-\infty}^{t} d \tau e^{i \omega \tau} \mathbf{E}(\tau)\right]+\text { c.c. }\right\}
\end{aligned}
$$

Here c.c. denotes the complex conjugate.

This object is now clearly a perfect time derivative to which the product rule has been applied, and so can be rewritten as

$$
\begin{aligned}
{\left[\frac{\partial}{\partial t} \mathbf{P}(t)\right]^{\dagger} \mathbf{E}(t)=} & \frac{\partial}{\partial t}\left\{\frac{1}{2 \pi} \int_{-\infty}^{+\infty} d \omega \| \hat{\alpha}_{E}(\omega)\right. \\
& \left.\times \int_{-\infty}^{t} d \tau e^{i \omega \tau} \mathbf{E}(\tau) \|^{2}\right\} .
\end{aligned}
$$

(Here the norm symbol $\|*\|$ indicates that one takes the length of its argument as a complex 3 vector.) This expression is manifestly the time derivative of a positive definite quadratic form in the electric field, albeit nonlocal in time. Repeating the above steps for the pair $(\mathbf{M}, \mathbf{H})$ we get an analogous formula,

$$
\begin{aligned}
\left(\frac{\partial}{\partial t} \mathbf{M}(t)\right)^{\dagger} \mathbf{H}(t)= & \frac{\partial}{\partial t}\left\{\frac{1}{2 \pi} \int_{-\infty}^{+\infty} d \omega \| \hat{\alpha}_{H}(\omega)\right. \\
& \left.\times \int_{-\infty}^{t} d \tau e^{i \omega \tau} \mathbf{H}(\tau) \|^{2}\right\} .
\end{aligned}
$$

We can now express the dispersive, dissipative version of Poynting's theorem (in the absence of macroscopic currents). Emphasizing the spatial dependencies heretofore suppressed, this conservation law is

$$
\frac{\partial u(\mathbf{x}, t)}{\partial t}+c \boldsymbol{\nabla} \cdot \mathbf{S}(\mathbf{x}, t)=0
$$

where the energy flux $\mathbf{S}(\mathbf{x}, t)$ is the usual Poynting vector,

$$
\mathbf{S}(\mathbf{x}, t)=\mathbf{E}(\mathbf{x}, t) \times \mathbf{H}(\mathbf{x}, t) .
$$

The total energy density $u(\mathbf{x}, t)$ is now somewhat more complicated than in the usual case, 


$$
\begin{aligned}
u(\mathbf{x}, t):= & \frac{1}{2}\|\mathbf{E}(\mathbf{x}, t)\|^{2}+\frac{1}{2}\|\mathbf{H}(\mathbf{x}, t)\|^{2} \\
& +\int_{-\infty}^{+\infty} d \omega\left[\left\|\hat{\alpha}_{E}(\mathbf{x}, \omega) \frac{1}{\sqrt{2 \pi}} \int_{-\infty}^{t} d \tau e^{i \omega \tau} \mathbf{E}(\mathbf{x}, \tau)\right\|^{2}\right. \\
& \left.+\left\|\hat{\alpha}_{H}(\mathbf{x}, \omega) \frac{1}{\sqrt{2 \pi}} \int_{-\infty}^{t} d \tau e^{i \omega \tau} \mathbf{H}(\mathbf{x}, \tau)\right\|^{2}\right]
\end{aligned}
$$

Here we remind the reader that over real frequencies the $\hat{\alpha}$ tensors are related to the susceptibilities and hence permittivity and permeability as follows:

$$
\begin{aligned}
\hat{\alpha}_{E}^{\dagger}(\mathbf{x}, \omega) \hat{\alpha}_{E}(\mathbf{x}, \omega) & =\omega \operatorname{Im}\left[\hat{\chi}_{E}(\mathbf{x}, \omega)\right]=\omega \operatorname{Im}[\hat{\boldsymbol{\epsilon}}(\mathbf{x}, \omega)-\hat{I}] \\
& =\omega \operatorname{Im}[\hat{\epsilon}(\mathbf{x}, \omega)], \\
\hat{\alpha}_{H}^{\dagger}(\mathbf{x}, \omega) \hat{\alpha}_{H}(\mathbf{x}, \omega) & =\omega \operatorname{Im}\left[\hat{\chi}_{H}(\mathbf{x}, \omega)\right]=\omega \operatorname{Im}[\hat{\mu}(\mathbf{x}, \omega)-\hat{I}] \\
& =\omega \operatorname{Im}[\hat{\mu}(\mathbf{x}, \omega)] .
\end{aligned}
$$

These last two formulas should also remind the reader that what is required in Eq. (50) is the imaginary parts of the spatially varying permittivity and permeability. Thus if, as in [14], composite media are considered, long range "effective" constitutive relations cannot be used to obtain Eq. (50), but rather recourse to the original, spatially resolved relations must be made. It is only the latter that are guaranteed to satisfy all the requirements of causality. In particular the effective constitutive parameters mentioned in Ref. [14] do not satisfy the high frequency asymptotics of causality (a) ensuring luminal front velocity. This does not mean that the composite media in such constructions are not causal (physically impossible), but only that the formulas for the effective constitutive relations are approximate, applying only for the low frequencies associated with the long range spatial averaging that give rise to such formulas (see also [15]).

We note that the expression for the current total dynamical energy density $u(\mathbf{x}, t)$ Eq. (50) contains the classical expression for the (heat) energy eventually dissipated to the medium. Due to propagation, we expect the fields to eventually vanish at any given position $\mathbf{x}$ as time $t \rightarrow \pm \infty$. Thus via Eq. (50) we expect the density of energy "left behind" (as $t \rightarrow+\infty)$ at any given position to be obtained only via the third, temporally nonlocal term,

$$
\begin{aligned}
u(\mathbf{x},+\infty)= & \int_{-\infty}^{+\infty} d \omega \omega\left[\mathbf{E}^{\dagger}(\mathbf{x}, \omega) \operatorname{Im}[\hat{\boldsymbol{\epsilon}}(\mathbf{x}, \omega)] \mathbf{E}(\mathbf{x}, \omega)\right. \\
& \left.+\mathbf{H}^{\dagger}(\mathbf{x}, \omega) \operatorname{Im}[\hat{\mu}(\mathbf{x}, \omega)] \mathbf{H}(\mathbf{x}, \omega)\right]
\end{aligned}
$$

This formula is the well known classical expression for the energy eventually dissipated to the medium [14].

\section{Discussion of the total dynamical energy density}

Definition (50) demonstrates that the density represented by $u$ in the conservation law Eq. (48) is a positive definite quadratic form in the fields. The positivity property is impor- tant to establish if $u$ is to be interpreted as a meaningful dynamical energy density that not only has the units of energy but can also prescribe the qualitative features of system dynamics. Such features include the boundedness (as well as existence and uniqueness) of solutions for all time, the asymptotic state of the solutions, and, since our dynamical equations (1) and (2) constitute a system of wave equations, the "domain of dependence" of solutions, i.e., the classical Sommerfeld-Brillouin result of vanishing of the fields outside the light cone of compactly supported initial data [4].

In another publication [3] we discuss in greater detail how the structure of dynamical energy density (50) suggests a mechanism for the Garrett and McCumber [6] and Chiao effects [7]. For now we limit our discussion to demonstrating that a causal medium responds to virtual frequencies and to giving a very geometric proof of the property of luminal front velocity. In addition, we discuss the connection between the dynamical energy density (50) and an approximate expression often employed.

\section{The medium responds to a virtual, instantaneous spectrum}

The form of Eq. (50) can be used to explain the phenomena by which the leading portion of an electromagnetic pulse exchanges energy with the causal medium differently than the trailing portion [3]. To see that this is possible, rewrite Eq. (50) as

$$
\begin{aligned}
u(\mathbf{x}, t):= & \frac{1}{2}\|\mathbf{E}(\mathbf{x}, t)\|^{2}+\frac{1}{2}\|\mathbf{H}(\mathbf{x}, t)\|^{2} \\
& +\int_{-\infty}^{+\infty} d \omega \omega\left[\mathbf{E}^{\dagger}(\mathbf{x}, \omega ; t) \operatorname{Im}[\hat{\boldsymbol{\epsilon}}(\mathbf{x}, \omega)] \mathbf{E}(\mathbf{x}, \omega ; t)\right. \\
& \left.+\mathbf{H}^{\dagger}(\mathbf{x}, \omega ; t) \operatorname{Im}[\hat{\mu}(\mathbf{x}, \omega)] \mathbf{H}(\mathbf{x}, \omega ; t)\right]
\end{aligned}
$$

where the instantaneous spectrum at time $t, \mathbf{F}(\mathbf{x}, \omega ; t)$, $(\mathbf{F}=\mathbf{E}$ or $\mathbf{H})$ is defined by

$$
\mathbf{F}(\mathbf{x}, \omega ; t):=\frac{1}{\sqrt{2 \pi}} \int_{-\infty}^{t} d \tau e^{i \omega \tau} \mathbf{F}(\mathbf{x}, \tau)
$$

The instantaneous spectrum $\mathbf{F}(\mathbf{x}, \omega ; t)$ is just the spectrum of a modified version of the "signal" $\mathbf{F}(\mathbf{x}, \tau)$ truncated or " turned off', at time $\tau=t$,

$$
\begin{array}{lll}
\mathbf{F}(\mathbf{x}, \tau) & ; \quad-\infty<\tau<t \\
\mathbf{0} & ; \quad t<\tau<+\infty
\end{array}
$$

[Note that in the limit $t \rightarrow \infty$, the instantaneous spectrum is simply the Fourier transform of $\mathbf{F}(\mathbf{x}, t)$ as per Eq. (7).]

That the energy density in a physical system must depend on the fields this way is made clear by causality: the energy at a given time $t$ cannot depend on future values of the fields producing it. It is also clear that the instantaneous spectra can be much broader at certain finite times than at its asymptotic $(t \rightarrow \infty)$ value. In particular it can be shown to be broadest at a given position $\mathbf{x}$ when the signal achieves its peak value there-i.e., when truncation produces the greatest disconti- 
nuity in the truncated signal (56). In this sense, we may say that the medium responds dynamically to "virtual" frequencies, i.e., to frequencies that would be produced if the signal were suddenly turned off. It is as if the causal medium must be prepared for this possibility and responds accordingly.

The instantaneous spectra contribute in Eq. (54) to the total energy density of the medium-field system through summation over all frequency contributions. Of course, the imaginary parts of the permittivity and permeability are also present in the integrand giving the energy density stored in the medium. The energy reactively stored in the dissipative medium is greatest, then, when the instantaneous spectrum produces the most overlap with the medium resonances, which resonances are given by peaks in the imaginary parts of (the eigenvalues of) the permittivity $\hat{\epsilon}$ and permeability $\hat{\mu}$. Depending on the detuning of the incident radiation from these resonances (i.e., depending on the asymptotic $t \rightarrow \infty$ value taken on by its instantaneous spectrum) this time of greatest energy storage can be before or after the peak of the propagating components of the pulse (which are given solely by the fields $\mathbf{E}$ and $\mathbf{H}$ ) have arrived at a specific position $\mathbf{x}$. This "temporal" disparity of energy storage in the medium (and subsequent retrieval from the medium) caused by the medium's response to virtual frequencies then leads to spatial redistribution of the field energy, giving rise to a (potentially anomalous) global energy transport mechanism.

It can be shown, though, that when this spatial redistribution of energy makes the pulse appear to move superluminally the redistribution does not constitute a signal in the direction of energy transport. Rather the redistribution is due to a change in the form of the energy - a change from medium to field energy, for example. Thus no matter how fast the pulse may appear to move in a global sense (e.g., in the sense of center of mass), the associated signal velocities are always luminal $[3,9]$. In this sense, the anomalous speeds apparently produced by these spatial redistributions are completely analogous to the phenomena in which two detectors can be made to "click" simultaneously regardless of their separation simply by irradiating them simultaneously with the same source. The clicking of the two detectors in this example does not, of course, constitute superluminal communication between those detectors, rather it merely constitutes simultaneous luminal communication between the source and the detectors.

\section{A dynamical energy density implies a maximum front speed}

In this section we show by looking at energy flow that the support of fields satisfying the Maxwell equations (1) and (2), with constitutive relations (3) and (4) prescribed by assumptions (a)-(c), can expand or contract no faster than $c$. The velocity of the support is called the front velocity. We begin by assuming that the total dynamical energy density $u$ as given by Eq. (50) is zero in some spherical region of space at a time $t_{i}$. We then demonstrate that this initial condition guarantees that $u$ is also zero on the space-time "cone" of slope $c$ with this initial sphere as its base (see Fig. 1). In other words, no energy (and hence no signal) can enter the initial sphere with a speed greater than $c$. (For a relevant similar derivation see [16].)

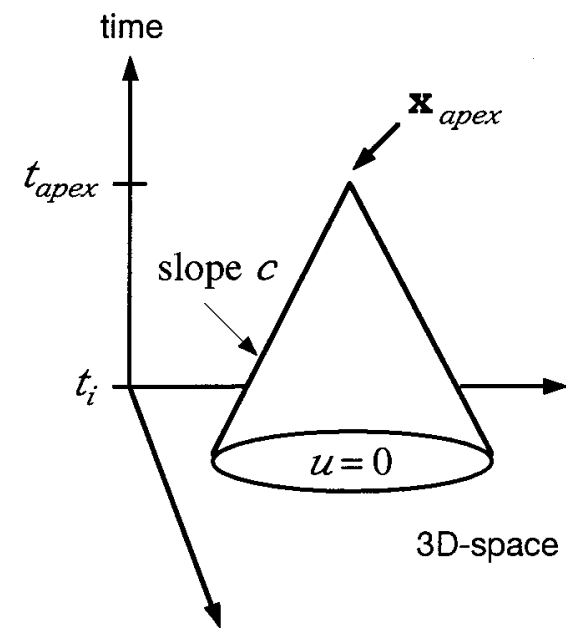

FIG. 1. The space-time "cone" of a spherical region of space that is initially free of energy. Three-dimensional space is represented by the horizontal dimensions and time proceeds vertically.

Given some final time $t_{\text {apex }}$, we prescribe an initial time $t_{i}\left(t_{i}<t_{\text {apex }}\right)$ at which $u$ vanishes inside an $\mathbf{x}$ ball of radius $c\left(t_{\text {apex }}-t_{i}\right)$ centered at position $\mathbf{x}_{\text {apex }}$ :

$$
u\left(\mathbf{x}, t_{i}\right)=0, \quad \mathbf{x} \in \mathcal{B}\left(\mathbf{x}_{\text {apex }}, c\left(t_{\text {apex }}-t_{i}\right)\right) .
$$

Here the notation is defined by

$$
\mathcal{B}\left(\mathbf{x}_{0}, r_{0}\right):=\left\{\mathbf{x} \mid\left\|\mathbf{x}-\mathbf{x}_{0}\right\| \leqslant r_{0}\right\} .
$$

[Note that in Fig. 1 the coordinates of the cone's apex are $\left(\mathbf{x}_{\text {apex }}, t_{\text {apex }}\right)$.] Given this initial state, we can now show that the energy density $u$, and thus the fields, vanish in the cone depicted in Fig. 1, i.e., in the forward light cone defined by

$$
\begin{aligned}
\mathcal{V}\left(\mathbf{x}_{\text {apex }}, t_{\text {apex }}\right) & :=\left\{(\mathbf{x}, t)\left\|\mathbf{x}-\mathbf{x}_{\text {apex }}\right\|\right. \\
& \left.\leqslant c\left(t_{\text {apex }}-t\right), t_{i} \leqslant t \leqslant t_{\text {apex }}\right\},
\end{aligned}
$$

thereby establishing luminal front velocity.

To this end consider the energy in the various $\mathbf{x}$ balls comprising the cone, one for each time $t$ in the cone. Let $\mathcal{E}_{\mathcal{V}}(t)$ denote these energies and note they are defined by

$$
\mathcal{E}_{\mathcal{V}}(t):=\int_{\mathcal{B}\left(\mathbf{x}_{\text {apex }}, c\left(t_{\text {apex }}-t\right)\right)} u(\mathbf{x}, t) d^{3} x ; \quad t_{i} \leqslant t \leqslant t_{\text {apex }} .
$$

Note also that since $u$ is positive definite, $\mathcal{E}_{\mathcal{V}}(t)$ is always non-negative,

$$
\mathcal{E}_{\mathcal{V}}(t) \geqslant 0 .
$$

Now from Eq. (57) we learn that $\mathcal{E}_{\mathcal{V}}(t)$ has the initial data

$$
\mathcal{E}_{\mathcal{V}}\left(t_{i}\right)=0 .
$$

We now show that $\mathcal{E}_{\mathcal{V}}(t)$ does not differ from this initial value for as long as it is defined, i.e., for all time $t$ in $\left[t_{i}, t_{\text {apex }}\right]$. Differentiating $\mathcal{E}_{\mathcal{V}}(t)$ [using Eq. (60)] we get 


$$
\begin{aligned}
\dot{\mathcal{E}}_{\mathcal{V}}(t)= & \int_{\mathcal{B}\left(\mathbf{x}_{\text {apex }}, c\left(t_{\text {apex }}-t\right)\right)} u_{t}(\mathbf{x}, t) d^{3} x \\
& -c \int_{\partial \mathcal{B}\left(\mathbf{x}_{\text {apex }}, c\left(t_{\text {apex }}-t\right)\right)} u(\mathbf{x}, t) d^{2} x ; \quad t_{i} \leqslant t \leqslant t_{\text {apex }} .
\end{aligned}
$$

The boundary term (the second integral) appears since the dimensions of the ball depend on time $t$. The $-c$ multiplying the boundary term appears since the ball's radius decreases in size as time proceeds forward, and does so at the rate $c$. The boundary $\partial \mathcal{B}\left[\mathbf{x}_{\text {apex }}, c\left(t_{\text {apex }}-t\right)\right]$ is the surface of the ball embedded at time $t$ (dimension 2).

Using the conservation law (48) to eliminate $u_{t}(\mathbf{x}, t)$ in the first integral, and then using the divergence theorem to exchange the volume integral for a surface integral, one gets that $\dot{\mathcal{E}}_{\mathcal{V}}(t)$ is determined by the values of certain quantities only on the ball's boundary,

$$
\begin{aligned}
\dot{\mathcal{E}}_{\mathcal{V}}(t)= & -c \int_{\partial \mathcal{B}\left(\mathbf{x}_{\text {apex }}, c\left(t_{\text {apex }}-t\right)\right)}[\mathbf{S}(\mathbf{x}, t) \cdot \mathbf{n}(\mathbf{x}) \\
& +u(\mathbf{x}, t)] d^{2} x ; \quad t_{i} \leqslant t \leqslant t_{\text {apex }} .
\end{aligned}
$$

Here $\mathbf{n}(\mathbf{x})$ is the unit outward normal to the boundary of the ball at position $\mathbf{x} \in \partial \mathcal{B}\left(\mathbf{x}_{\text {apex }}, c\left(t_{\text {apex }}-t\right)\right)$. In Sec. III we show that $|u(\mathbf{x}, t)| \geqslant\|\mathbf{S}(\mathbf{x}, t)\|$. Since $u$ is positive definite, this establishes the more useful fact that

$u(\mathbf{x}, t)=|u(\mathbf{x}, t)| \geqslant\|\mathbf{S}(\mathbf{x}, t)\| \geqslant|\mathbf{S}(\mathbf{x}, t) \cdot \mathbf{n}(\mathbf{x})| \geqslant-\mathbf{S}(\mathbf{x}, t) \cdot \mathbf{n}(\mathbf{x})$,

i.e.,

$$
\mathbf{S}(\mathbf{x}, t) \cdot \mathbf{n}(\mathbf{x})+u(\mathbf{x}, t) \geqslant 0 .
$$

Thus the integrand in Eq. (64) is non-negative and so the energy does not increase,

$$
\dot{\mathcal{E}}_{\mathcal{V}}(t) \leqslant 0 ; \quad t_{i} \leqslant t \leqslant t_{\text {apex }} .
$$

Equation (67) together with the initial data (62) demands that

$$
\mathcal{E}_{\mathcal{V}}(t) \leqslant \mathcal{E}_{\mathcal{V}}\left(t_{i}\right)=0 ; \quad t_{i} \leqslant t \leqslant t_{\text {apex }},
$$

which contradicts the non-negativity of $\mathcal{E}_{\mathcal{V}}(t)$ Eq. (61) unless

$$
\mathcal{E}_{\mathcal{V}}(t)=\mathcal{E}_{\mathcal{V}}\left(t_{i}\right)=0 ; \quad t_{i} \leqslant t \leqslant t_{\text {apex }} .
$$

Since $u$ is positive definite in the fields $\mathbf{E}(\mathbf{x}, t)$ and $\mathbf{H}(\mathbf{x}, t), \mathcal{E}_{\mathcal{V}}(t)$ vanishes for time in the indicated interval only if those fields vanish in the cone $\mathcal{V}\left(\mathbf{x}_{\text {apex }}, t_{\text {apex }}\right)$. This together with the causal relationship of the other two fields to these fields then demands that all four fields vanish in the cone, thereby establishing luminal front velocity.

\section{The relationship between the dynamical energy and the traditional approximate kinematic energy}

From definition (50) it is clear that the dynamical energy density differs from the approximate energy density,

$$
u_{\text {approx }}(\mathbf{x}, t):=\frac{1}{2} \mathbf{E}(\mathbf{x}, t) \cdot \mathbf{D}(\mathbf{x}, t)+\frac{1}{2} \mathbf{B}(\mathbf{x}, t) \cdot \mathbf{H}(\mathbf{x}, t) .
$$

Some texts on classical electrodynamics originally identified Eq. (70) as the total energy density, i.e., as the object conserved in Poynting's theorem (48). Subsequent editions have clarified that the quantity (70) is valid only for the time average of a single frequency. However, they have not demonstrated that the correct object to be considered is a dynamical total energy density (i.e., a positive definite form indicating the closed nature of the dynamics).

To make a comparison with Eq. (50), we eliminate $\mathbf{D}(\mathbf{x}, t)$ and $\mathbf{B}(\mathbf{x}, t)$ from the expression by way of Eq. (22), (39) (without the time derivatives), and (34) (and the "magnetic", analogs of these relations). Writing the result as closely as possible to the form of Eq. (50), we get

$$
\begin{aligned}
u_{\text {approx }}(\mathbf{x}, t) & \frac{1}{2}\|\mathbf{E}(\mathbf{x}, t)\|^{2}+\frac{1}{2}\|\mathbf{H}(\mathbf{x}, t)\|^{2} \\
& +\frac{i}{4 \pi} \int_{-\infty}^{+\infty} d \omega\left[\mathbf{E}^{\dagger}(\mathbf{x}, t) e^{-i \omega t} \frac{\hat{\alpha}_{E}^{\dagger}(\mathbf{x}, \omega) \hat{\alpha}_{E}(\mathbf{x}, \omega)}{\omega}\right. \\
& \times \int_{-\infty}^{t} d \tau e^{i \omega \tau} \mathbf{E}(\mathbf{x}, \tau) \\
& +\mathbf{H}^{\dagger}(\mathbf{x}, t) e^{-i \omega t} \frac{\hat{\alpha}_{H}^{\dagger}(\mathbf{x}, \omega) \hat{\alpha}_{H}(\mathbf{x}, \omega)}{\omega}
\end{aligned}
$$$$
\left.\times \int_{-\infty}^{t} d \tau e^{i \omega \tau} \mathbf{H}(\mathbf{x}, \tau)\right]+ \text { c.c. }
$$

Clearly densities (50) and (71) constitute different quadratic forms in the fields. In particular, whereas the dynamic total energy density (50) is manifestly positive definite for any field history, the approximate total energy density (71) can be shown to alternate sign for certain physically relevant examples.

To illustrate this effect, we here consider the simple case of monochromatic electric fields given by

$$
\mathbf{E}(\mathbf{x}, t)=\mathbf{E}_{0}(\mathbf{x}) e^{-i \Omega t}+\text { c.c. }
$$

(In this example we examine only the electric contribution.) Also, it is useful to recall the distributional identities

$$
\begin{aligned}
e^{-i\left(\omega-\omega^{\prime}\right) t} \int_{-\infty}^{t} d \tau e^{i\left(\omega-\omega^{\prime}\right) \tau} & =\lim _{\epsilon \rightarrow 0^{+}} \frac{1}{\epsilon+i\left(\omega-\omega^{\prime}\right)} \\
& =\pi \delta\left(\omega-\omega^{\prime}\right)-i P\left(\frac{1}{\omega-\omega^{\prime}}\right) .
\end{aligned}
$$


Here $\mathrm{P}\left[1 /\left(\omega-\omega^{\prime}\right)\right]$ indicates the Cauchy Principal value distribution centered at $\omega=\omega^{\prime}$, and $\delta\left(\omega-\omega^{\prime}\right)$ indicates the Dirac $\delta$ function (distribution) also centered at $\omega=\omega^{\prime}$. Inserting the fields (72) into (71), using the relevant versions of the distributional identities (73), and finally the KramersKronig relations (32) in reverse, we get (simplifying to the isotropic case)

$$
\begin{gathered}
u_{\text {approx }}(\mathbf{x}, t)=\left\|\mathbf{E}_{0}(\mathbf{x})\right\|^{2} \operatorname{Re}[\hat{\boldsymbol{\epsilon}}(\Omega)]+\frac{\mathbf{E}_{0}^{T}(\mathbf{x}) \mathbf{E}_{0}(\mathbf{x})}{2} e^{-2 i \Omega t} \hat{\boldsymbol{\epsilon}}(\Omega) \\
+\frac{\mathbf{E}_{0}^{\dagger}(\mathbf{x}) \mathbf{E}_{0}^{*}(\mathbf{x})}{2} e^{+2 i \Omega t} \hat{\boldsymbol{\epsilon}}^{*}(\Omega)
\end{gathered}
$$

We investigate the two extreme values of Eq. (74) (at each point $\mathbf{x}$ ) by limiting Eq. (74) to the set of times at which the kinematic density is stationary. At those times (denoted $\bar{t}$ ) we find that

$$
\frac{\mathbf{E}_{0}^{\dagger}(\mathbf{x}) \mathbf{E}_{0}^{*}(\mathbf{x})}{2} e^{+2 i \Omega \bar{t}} \hat{\epsilon}^{*}(\Omega)=\frac{\mathbf{E}_{0}^{T}(\mathbf{x}) \mathbf{E}_{0}(\mathbf{x})}{2} e^{-2 i \Omega \bar{t}} \hat{\boldsymbol{\epsilon}}(\Omega) .
$$

Note that both of these quantities are real at those times. The extreme values of the density (74) are then

$$
\begin{aligned}
u_{\text {approx }}(\mathbf{x}, \bar{t})= & \left\|\mathbf{E}_{0}(\mathbf{x})\right\|^{2} \operatorname{Re}[\hat{\boldsymbol{\epsilon}}(\Omega)] \\
& +\mathbf{E}_{0}^{T}(\mathbf{x}) \mathbf{E}_{0}(\mathbf{x}) e^{-2 i \Omega} \hat{t} \hat{\boldsymbol{\epsilon}}(\Omega) .
\end{aligned}
$$

Using the fact that the second quantity in Eq. (76) is real at these times, we realize that

$$
e^{-2 i \Omega \bar{t}}= \pm \frac{\mathbf{E}_{0}^{\dagger}(\mathbf{x}) \mathbf{E}_{0}^{*}(\mathbf{x}) \hat{\epsilon}^{*}(\Omega)}{\left|\mathbf{E}_{0}^{T}(\mathbf{x}) \mathbf{E}_{0}(\mathbf{x}) \hat{\boldsymbol{\epsilon}}(\Omega)\right|}
$$

in which case (76) becomes (after simplification)

$$
u_{\text {approx }}(\mathbf{x}, \bar{t})=\left\|\mathbf{E}_{0}(\mathbf{x})\right\|^{2}\{\operatorname{Re}[\hat{\epsilon}(\Omega)] \pm|\hat{\epsilon}(\Omega)|\} .
$$

In Eq. (78) it is now clear that the approximate density does not have definite sign so long as $\operatorname{Im}[\hat{\epsilon}(\Omega)]$ is not zero.

Note that in the limit of static fields, however, the dynamical and approximate results agree: Using real symmetry, whereby $\operatorname{Im}[\hat{\epsilon}(\Omega)]$ goes to zero when $\Omega$ does, we see that Eq. (74) becomes (after some simplification)

$u_{\text {approx }}(\mathbf{x}, t)=\frac{\operatorname{Re}[\hat{\boldsymbol{\epsilon}}(0)]}{2}\left\|\mathbf{E}_{0}(\mathbf{x})+\mathbf{E}_{0}^{*}(\mathbf{x})\right\|^{2}=\frac{\hat{\epsilon}(0)}{2}\|\mathbf{E}(\mathbf{x}, t)\|^{2}$,

as expected. Using identities (73) the dynamical energy density (50) becomes, for the fields given in Eq. (72) at $\Omega=0$,

$$
\begin{aligned}
u(\mathbf{x}, t) & =\frac{1}{2}\left\|\mathbf{E}_{0}(\mathbf{x})+\mathbf{E}_{0}^{*}(\mathbf{x})\right\|^{2}\left(1+\frac{1}{\pi} \mathrm{P} \int_{-\infty}^{+\infty} d \omega \frac{\operatorname{Im}[\hat{\boldsymbol{\epsilon}}(\omega)]}{\omega-0}\right) \\
& =\frac{1}{2}\left\|\mathbf{E}_{0}(\mathbf{x})+\mathbf{E}_{0}^{*}(\mathbf{x})\right\|^{2} \operatorname{Re}[\hat{\boldsymbol{\epsilon}}(0)] \\
& =\frac{\hat{\boldsymbol{\epsilon}}(0)}{2}\|\mathbf{E}(\mathbf{x}, t)\|^{2}
\end{aligned}
$$

the second equality following from Kramers-Kronig for this isotropic case.

\section{GLOBAL ENERGY TRANSPORT VELOCITY}

In previous work, we investigated a certain "temporal center-of-mass" of an electromagnetic pulse [5]. We found, among other things, that this formalism provided a framework wherein the classical notion of group velocity was meaningful even for broad-band pulses. The following represents the spatial analog of that work. As is evidenced by the weight of recent works on superluminal electromagnetic phenomena (for a "small" sampling see [17]), this issue of the nature of global energy transport that we and others have addressed is clearly not the local one addressed by the (otherwise very satisfying) classical Sommerfeld-Brillouin result. Nevertheless, in the following one will see that, in contrast to the "temporally oriented" view of the properties of global total energy transport reported in [5], the "spatially oriented" view is very much a global generalization of Sommerfeld and Brillouin's local result.

We begin by defining the position of the total dynamical energy as the normalized, first spatial moment of the total dynamical energy density,

$$
\mathbf{x}_{u}(t):=\frac{\int d^{3} x \mathbf{x} u(\mathbf{x}, t)}{\int d^{3} x u(\mathbf{x}, t)}
$$

$$
=\mathcal{E}^{-1} \int d^{3} x \mathbf{x} u(\mathbf{x}, t)
$$

The integrals are over all space and we have defined the total energy

$$
\mathcal{E}:=\int d^{3} x u(\mathbf{x}, t)
$$

Having defined the position of the total energy $\mathbf{x}_{u}(t)$, we then define the velocity of the total energy $\mathbf{v}_{u}(t)$ in the natural way, i.e., by time differentiation of the position

$$
\mathbf{v}_{u}(t):=\frac{d \mathbf{x}_{u}(t)}{d t} .
$$

Making use of the definition of the position (82) and by use of Poynting's conservation law (48) we find that 


$$
\begin{aligned}
\mathbf{v}_{u}(t) & =\mathcal{E}^{-1} \int d^{3} x \mathbf{x} \frac{\partial u(\mathbf{x}, t)}{\partial t} \\
& =-c \mathcal{E}^{-1} \int d^{3} x \mathbf{x} \boldsymbol{\nabla} \cdot \mathbf{S}(\mathbf{x}, t) .
\end{aligned}
$$

Integration by parts then gives

$$
\mathbf{v}_{u}(t)=c \mathcal{E}^{-1} \int d^{3} x \mathbf{S}(\mathbf{x}, t) .
$$

The fact that the magnitude of the velocity so defined is always bounded by $c$ is now straightforward. Ostensibly it amounts to no more than a statement of the fact that the magnitude of the Poynting vector $\mathbf{S}(\mathbf{x}, t)=\mathbf{E}(\mathbf{x}, t) \times \mathbf{H}(\mathbf{x}, t)$ is always less than or equal to the energy density $u(\mathbf{x}, t)$,

$$
\begin{aligned}
\left\|\mathbf{v}_{u}(t)\right\| & =c \mathcal{E}^{-1}\left\|\int d^{3} x \mathbf{E}(\mathbf{x}, t) \times \mathbf{H}(\mathbf{x}, t)\right\| \\
& \leqslant c \mathcal{E}^{-1} \int d^{3} x\|\mathbf{E}(\mathbf{x}, t) \times \mathbf{H}(\mathbf{x}, t)\| \\
& \leqslant c \mathcal{E}^{-1} \int d^{3} x\left\{\frac{1}{2}\|\mathbf{E}(\mathbf{x}, t)\|^{2}+\frac{1}{2}\|\mathbf{H}(\mathbf{x}, t)\|^{2}\right\} \\
& \leqslant c \mathcal{E}^{-1} \int d^{3} x u(\mathbf{x}, t) \\
& =c \mathcal{E}^{-1} \mathcal{E}=c .
\end{aligned}
$$

In passing from Eq. (89) to Eq. (90) we used Lagrange's identity, and in passing from Eq. (90) to Eq. (91) we used the definition of the total dynamical energy density $u(\mathbf{x}, t)$, Eq. (50).

Lastly we show that the total dynamical energy's centerof-mass velocity just derived is a spatial average of the traditional energy transport velocity. Denote and define the " $u$-average" of a measurable $\mathcal{O}(\mathbf{x}, t)$ by

$$
\langle\mathcal{O}(\mathbf{x}, t)\rangle_{u}:=\frac{\int d^{3} x \mathcal{O}(\mathbf{x}, t) u(\mathbf{x}, t)}{\int d^{3} x u(\mathbf{x}, t)} .
$$

Then, with this notation, we see that

$$
\mathbf{v}_{u}(t)=\left\langle\left(c \frac{\mathbf{S}}{u}\right)(\mathbf{x}, t)\right\rangle_{u}=\left\langle\mathbf{v}_{\mathcal{E}}(\mathbf{x}, t)\right\rangle_{u},
$$

where $\mathbf{v}_{\mathcal{E}}(\mathbf{x}, t)$ is the traditional energy transport velocity. Note that in Eqs. (88) through (91) we also effectively demonstrated that the traditional energy transport velocity is luminal for passive dielectrics,

$$
\left\|\mathbf{v}_{\mathcal{E}}(\mathbf{x}, t)\right\| \leqslant c .
$$

By more complicated arguments, in Ref. [3] we also show that the same is true for active dielectrics.

\section{SUPERLUMINAL GLOBAL TRANSPORT OF SUBSETS OF THE TOTAL ENERGY: THE LORENTZ MODEL}

While the global notion of energy transport defined by center-of-mass motion of the total dynamical energy in a passive media is always luminal, global energy transport is not so constrained when only a subset of the total dynamical energy is considered. This indicates that in the global sense, the root of superluminal behavior is associated with incomplete energy accounting. (Note that via the SommerfeldBrillouin theorems, it is only in a nonlocal sense that superluminal phenomena are not strictly prohibited.)

In order to simplify the discussion, we consider the Abraham-Lorentz model of a nonmagnetic $[H(x, t)$ $=B(x, t)]$ homogeneous, isotropic dielectric with a single resonance frequency, and consider only one-dimensional solutions of the original three-dimensional system. In one space dimension, we can write the equations as a system of first order partial differential equations,

$$
\begin{aligned}
\frac{\partial}{\partial t}\left(\begin{array}{c}
E \\
B \\
P \\
Q
\end{array}\right)(x, t)=\frac{\partial}{\partial x}\left(\begin{array}{cccc}
0 & -c & 0 & 0 \\
-c & 0 & 0 & 0 \\
0 & 0 & 0 & 0 \\
0 & 0 & 0 & 0
\end{array}\right)\left(\begin{array}{l}
E \\
B \\
P \\
Q
\end{array}\right)(x, t) \\
+\left(\begin{array}{cccc}
0 & 0 & 0 & -\omega_{p} \\
0 & 0 & 0 & 0 \\
0 & 0 & 0 & \omega_{0} \\
\omega_{p} & 0 & -\omega_{0} & -\gamma
\end{array}\right)\left(\begin{array}{c}
E \\
B \\
P \\
Q
\end{array}\right)(x, t) .
\end{aligned}
$$

We note in passing that since the eigenvalues of the first matrix on the right of equation (96) (less the spatial derivative) are real, the system is hyperbolic. Furthermore, the theory of hyperbolic partial differential equations dictates that these eigenvalues give the limiting speeds at which singularities propagate so that for this model we already have the (luminal) Sommerfeld-Brillouin result for the front velocity [18].

The scalar permittivity $\epsilon(\omega)$ for this model can be calculated to be the usual prototypical example [10] possessing all of the relevant requirements of causality and passivity,

$$
\epsilon(\omega)=1+\frac{\omega_{p}^{2}}{-\omega^{2}-i \gamma \omega+\omega_{0}^{2}}
$$

Using the fact that the operator on the right of Eq. (96) is already in a form in which it can be written as a sum of an operator that is skew symmetric and one that is negative definite with respect to the usual inner product, we see that Eq. (96) dictates a law of dissipation [similar to the law of conservation (48)] simply by expressing the time evolution of the particular positive definite quadratic form associated with the (relevant) identity matrix, 


$$
\begin{gathered}
\left(\begin{array}{ll}
E B & P Q
\end{array}\right)\left(\begin{array}{cccc}
1 & 0 & 0 & 0 \\
0 & 1 & 0 & 0 \\
0 & 0 & 1 & 0 \\
0 & 0 & 0 & 1
\end{array}\right)\left(\begin{array}{c}
E \\
B \\
P \\
Q
\end{array}\right)(x, t) \\
=\left(E^{2}+B^{2}+P^{2}+Q^{2}\right)(x, t)
\end{gathered}
$$

We will denote this form (divided by 2 ) by $u_{R}(x, t)$ and, in order to distinguish it from the total energy density that obeys a conservative law, will call it the free-energy density (or distribution). Though we do not engage in a statistical mechanics treatment, we justify the use of this term as follows. We can interpret Eq. (96) as a phenomenological description of a system in which $u_{R}(x, t)$ has the interpretation of being the sum of the densities associated with energy stored in the macroscopic fields and that stored in the coherent motions of the molecular dipoles of the dispersive medium (mechanical energy density). In this phenomenological treatment, clearly $u_{R}(x, t)$ cannot be interpreted as containing energy deposited irreversibly in the medium via incoherent motions and their associated degrees of freedom.

The law of dissipation associated with this particular quadratic form, or energy projection, is

$$
\frac{\partial}{\partial t}\left(\frac{1}{2} E^{2}+\frac{1}{2} B^{2}+\frac{1}{2} P^{2}+\frac{1}{2} Q^{2}\right)+\frac{\partial}{\partial x}(c E B)=-2 \gamma\left(\frac{1}{2} Q^{2}\right) .
$$

Here we have suppressed the coordinates $x$ and $t$.

Note that if we define the free-energy density's velocity to be the ratio of the (suitably averaged) flux $c E B$ to the (similarly averaged) density $u_{R}=\left(\frac{1}{2} E^{2}+\frac{1}{2} B^{2}+\frac{1}{2} P^{2}+\frac{1}{2} Q^{2}\right)$, we are guaranteed to get a luminal result since

$$
\frac{|c E B|}{u_{R}} \leqslant c .
$$

Unfortunately this definition of the velocity generically has almost nothing to do with the gross motion of the freeenergy distribution $u_{R}$ because the evolution of the free energy is dissipative. If one simply views the results of a numerical simulation of Eq. (96) by watching a movie of $u_{R}(x, t)$ passing by, the perceived speed of the pulse can be arbitrarily large, depending on the system preparation. In the following, we make this observation concrete by showing analytically that a pulse's free energy "center-of-mass", $x_{u_{R}}(t)$, defined as

$$
x_{u_{R}}(t):=\frac{\int d x \times u_{R}(x, t)}{\int d x u_{R}(x, t)},
$$

(with the integration over all $x$ ) can move with any speed.

The velocity corresponding to this definition of position is obtained by time differentiating the center of mass. We use the law of transport (99) to eliminate the time derivative of the density $u_{R}$, and then use integration by parts to eliminate spatial derivatives on the flux $E B$. After some simplification we obtain

$$
\begin{aligned}
v_{u_{R}}(t)= & c \frac{\int d x E(x, t) B(x, t)}{\int d x u_{R}(x, t)}+\gamma\left\{\left(\frac{\int d x Q^{2}(x, t)}{\int d x u_{R}(x, t)}\right)\right. \\
& \times\left[\frac{\int d x x \bar{u}_{R}(x, t)}{\int d x u_{R}(x, t)}\right]-\left(\frac{\int d x \bar{u}_{R}(x, t)}{\int d x u_{R}(x, t)}\right) \\
& \left.\times\left[\frac{\int d x x Q^{2}(x, t)}{\int d x u_{R}(x, t)}\right]\right\}
\end{aligned}
$$

where

$$
\bar{u}_{R}:=u_{R}-\frac{1}{2} Q^{2}=\frac{1}{2} E^{2}+\frac{1}{2} B^{2}+\frac{1}{2} P^{2} .
$$

In Eq. (102) the velocity is expressed as having two components, the first not dependent on system parameters explicitly and the second explicitly dependent upon the damping rate $\gamma$. For each time $t$ the two terms are functionals of functions of position $x$. The first functional can be shown to possess extrema $\pm c$. This is done by showing that the absolute value of the integrand of its numerator never exceeds (but can be equal to) the integrand of its denominator (which is non-negative) multiplied by $c$. Below we will show that the second term, which is multiplied by the damping rate $\gamma$, is an unbounded functional in a relevant function space. Thus, in this function space, the center-of-mass velocity functional is unbounded when damping is present but is bounded luminally when damping is absent. Note that we do not presently address the issue of the duration of superluminal behavior in the free-energy's center-of-mass motion, but only the issue of superluminal system preparations, i.e., of whether the system can, in principle, be initially prepared so as to demonstrate superluminal behavior in the motion of the free-energy center of mass.

To that end we consider the velocity at $t=0$, and consider a two-parameter family of system preparations, all members of which correspond to the same initial free energy. We then show that, when damping is present, the initial center-ofmass velocity increases without bound as the difference in the two parameters increase. In order to motivate how this is accomplished we pause to comment on which details of the structure of the second functional in Eq. (102) suggest that this can be done. We have tried to make this structure evident by parallel uses of parenthesis and square brackets. The two terms in large square brackets have units of position. The left term in square brackets measures the center of mass (as normalized by the free energy) of the energy stored in the fields and in the displacements of the dipoles from equilibrium (dipole potential energy). The right term in square 
brackets measures the center of mass (as normalized by the free energy) of the kinetic energy associated with the motion of the dipoles. Note that the two energy projections just discussed sum to the free energy. Consequently we will speak of them as being compliments with respect to the free energy. In contrast to the terms measuring positions, the two terms in large parenthesis are homogeneous and so are unitless. They measure the relative amounts of energy associated with the two centers of mass just described. As such they may be described as weights for the associated "masses." Note that the weights are paired (via multiplication) with their complement centers. Thus, since the notion of complement is with respect to the free energy, and since the free energy is the distribution used to normalize the two centers of mass, the two products of these four objects give the centers of reduced mass of the two complimentary energy projections. Thus we see that the second functional in Eq. (102) gives a measure of the difference in the centers of reduced mass of the kinetic energy and its free-energy complement. Thus the "additional" velocity of the free-energy distribution (i.e., the component of its velocity that is attributable to damping) is just the damping rate multiplied by a difference in (representative) positions of two complementary distributions.

To make these connections more obvious we introduce some notation. Relabel the kinetic energy density $Q^{2}(x, t) / 2$ as $m_{1}^{t}(x)$ and relabel its complement $\bar{u}_{R}(x, t)=u_{R}(x, t)$ $-Q^{2}(x, t) / 2$ as $m_{2}^{t}(x)$. Also relabel the corresponding energies (the integrals of the densities) by the same symbols but with capital letters and, of course, without reference to the position $x$,

$$
\begin{gathered}
M_{1}^{t}:=\int d x m_{1}^{t}(x), \quad \text { and } \\
M_{2}^{t}:=\int d x m_{2}^{t}(x) .
\end{gathered}
$$

Then the center-of-mass velocity of the free energy can be expressed as

$$
\begin{aligned}
v_{u_{R}}(t)= & c \frac{\int d x E(x, t) B(x, t)}{\int d x u_{R}(x, t)}+2 \gamma\left\{\frac{\int d x \times \frac{M_{1}^{t} m_{2}^{t}(x)}{M_{1}^{t}+M_{2}^{t}}}{M_{1}^{t}+M_{2}^{t}}\right. \\
& \left.-\frac{\int d x x \frac{m_{1}^{t}(x) M_{2}^{t}}{M_{1}^{t}+M_{2}^{t}}}{M_{1}^{t}+M_{2}^{t}}\right\} .
\end{aligned}
$$

Here we see that the integrands used to define the centers of mass appear analogous to the classical expression for reduced mass in the two-body problem.

We now consider the velocity at $t=0$ and a corresponding class of system preparations or initial conditions that show that the velocity at this instant can be arbitrarily large if and only if $\gamma$ does not vanish. To find the simplest expression for this initial velocity we choose initial data for which the total initial kinetic energy is equal to its complement. On the other hand, if we choose the associated distributions to be identical then the differences in the associated centers of (reduced) mass will be zero. The simplest way to get a nontrivial and interesting result is to choose the two distributions to be translates of each other: For some even and square integrable function $f(x)$ different from 0 and for some positions $x_{1}$ and $x_{2}$ define $m_{1}^{0}(x)$ and $m_{2}^{0}(x)$ by

$$
\begin{gathered}
m_{1}^{0}(x):=f^{2}\left(x-x_{1}\right), \quad \text { and } \\
m_{2}^{0}(x):=f^{2}\left(x-x_{2}\right) .
\end{gathered}
$$

Then we get $M_{1}^{0}=M_{2}^{0}=\int d x f^{2}(x)=M>0$, and then

$$
\int d x \times m_{i}(x)=x_{i} M, \quad i=1,2 .
$$

Then Eq. (106) reduces at $t=0$ to

$$
v_{u_{R}}(0)=c \frac{\int d x E(x, 0) B(x, 0)}{\int d x u_{R}(x, 0)}+\frac{\gamma}{2}\left(x_{2}-x_{1}\right) .
$$

In order to make this expression more explicit we can, for example, further decompose the initial energy densities as follows. Choose $E(x, 0)=B(x, 0)=f\left(x-x_{2}\right)$ and $P(x, 0)=0$ [and $Q(x, 0)=\sqrt{2} f\left(x-x_{1}\right)$ ]. With this two-parameter family of choices for the initial data, the initial velocity of the free energy center of mass (110) reduces to

$$
v_{u_{R}}(0)=\frac{c}{2}+\frac{\gamma}{2} \Delta x
$$

(Here we have expressed the difference in the centers of reduced mass of the kinetic energy and its complement, $x_{2}$ $-x_{1}$, as $\Delta x$.) It now becomes clear that if $\gamma$ is not zero, the speed of the free-energy center of mass can be increased arbitrarily, as long as the "position" of the kinetic energy distribution can be made to lead (or lag) that of its free energy complement by arbitrarily large distances. Furthermore this notion of position becomes more natural and precise as the variance of $f(x)$ reduces. In fact there is nothing to keep us from considering the limit in which $f(x) \rightarrow \sqrt{\delta(x)}$ : in this limit we still get the (not luminal) result (111) for the initial center-of-mass velocity of the free energy.

\section{SUMMARY}

In this paper the luminality of both local and global notions of total energy transport in very general (anisotropic, inhomogeneous, passive) media was established. In lieu of specific microscopic models, these results were established using only the macroscopic limitations of causality and passivity. Specifically these estimates were obtained by (1) developing total dynamical energy densities for these media (i.e. conserved, positive definite, quadratic forms) and then by (2) considering the time evolution of their associated fi- 
nite and infinite volume energies, respectively. We also showed how a total dynamical energy density demonstrates that a causal media responds to a virtual, instantaneous field spectrum weighted by system resonances, that this density gives the heat energy eventually dissipated to the medium, but that this density is not the same as the one derived from kinematic arguments.

The connection between the local concept of energy transport velocity and the global concept of total energy centerof-mass velocity was also established, and the importance of avoiding subsets of a total energy (i.e., forms that are not conserved) in establishing the global notion of luminality was emphasized. For the Lorentz model (a specific example of the general media considered at the onset), a certain subset of the total energy was shown to have the potential for demonstrating what appears (in the center of mass picture) to be an arbitrarily fast global energy transport mechanism. It was emphasized, however, that this appearance of high velocity does not constitute energy transport from one detector to another, but simply indicates that other energy already downstream has been converted to the type being observed. This illustrates the general principle that classical "superluminal" effects are intimately linked with an incomplete energy accounting.

\section{APPENDIX: KINETIC SYMMETRY AND PASSIVITY IMPLY DISSIPATION}

We prove (c) and (b) $\Rightarrow \mathrm{c}^{\prime}$ for $\hat{\epsilon}$. That the converse $\left(\mathrm{c}^{\prime}\right)$ and $(b) \Rightarrow c$ ) is false (without invoking more structure) is verified by specific counter examples. However, as is discussed in the main text, if we invoke the structure that the eigenvectors of the tensors can be taken as real, it is then true that $\left(c^{\prime}\right)$ and $(b) \Rightarrow(c)$. Physically this extra structure means that the eigenvectors of the tensors can correspond to bonafide directions in real 3 space, the principal axes, say, of a crystal. Otherwise these eigenvectors correspond to "directions" only in complex 3 space, but whose real and imaginary parts in real 3 space can be made to correspond to directions together with rotation angles.

In order to avoid needless repetition, we state here once that in the following paragraph all statements about the permittivity tensor $\hat{\boldsymbol{\epsilon}}$ are valid when it is evaluated at real positive frequencies $\omega$, i.e., when the restrictions of assumption (c) are enforced.
Let $\mathbf{v}_{\lambda}$ be an eigenvector of $\hat{\epsilon}$ for eigenvalue $\lambda \in \rho$. The eigenvalue can be expressed in terms of the eigenvector and the real and imaginary parts of $\hat{\epsilon}$ via the formula

$$
\lambda=\frac{\mathbf{v}_{\lambda}^{\dagger} \operatorname{Re}[\hat{\epsilon}] \mathbf{v}_{\lambda}}{\mathbf{v}_{\lambda}^{\dagger} \mathbf{v}_{\lambda}}+i \frac{\mathbf{v}_{\lambda}^{\dagger} \operatorname{Im}[\hat{\epsilon}] \mathbf{v}_{\lambda}}{\mathbf{v}_{\lambda}^{\dagger} \mathbf{v}_{\lambda}} .
$$

The kinetic symmetry (b) of $\hat{\epsilon}$ implies this symmetry of its real and imaginary parts, which, since they are each real, shows that they are each trivially Hermitian. Taking the Hermitian conjugate, then, of our formula, and using the Hermitian properties (just established) of the real and imaginary parts of $\hat{\epsilon}$ shows that the imaginary part of $\lambda$ can be expressed as

$$
\operatorname{Im}[\lambda]=\frac{\lambda-\lambda^{\dagger}}{2 i}=\frac{\mathbf{v}_{\lambda}^{\dagger} \operatorname{Im}[\hat{\epsilon}] \mathbf{v}_{\lambda}}{\mathbf{v}_{\lambda}^{\dagger} \mathbf{v}_{\lambda}}
$$

At this point it is already obvious that $\operatorname{Im}[\lambda]>0$ since $\operatorname{Im}[\hat{\epsilon}]$ is positive definite, but we make the proof even more explicit since we will use a certain notation that these details provide in the main text (Sec. II B) where we derive the total energy of the system: since we assume (c), i.e. since we assume that $\operatorname{Im}[\hat{\epsilon}]$ is a positive definite tensor, and since, as we have just shown, $\operatorname{Im}[\hat{\epsilon}]$ is trivially Hermitian, this tensor can be factored and expressed as

$$
\operatorname{Im}[\hat{\epsilon}]=\hat{\beta}^{\dagger} \hat{\beta}
$$

so that the imaginary part of $\lambda$ is then expressed as

$$
\operatorname{Im}[\lambda]=\frac{\mathbf{v}_{\lambda}^{\dagger} \hat{\beta}^{\dagger} \hat{\beta} \mathbf{v}_{\lambda}}{\mathbf{v}_{\lambda}^{\dagger} \mathbf{v}_{\lambda}}=\frac{\left\|\hat{\beta} \mathbf{v}_{\lambda}\right\|^{2}}{\left\|\mathbf{v}_{\lambda}\right\|^{2}} \geqslant 0,
$$

regardless of the relationship of the eigenvector $\mathbf{v}_{\lambda}$ of $\hat{\epsilon}$ to the tensor $\operatorname{Im}[\hat{\epsilon}]$. However, since equality is achieved only if $\mathbf{v}_{\lambda}$ happens to be a nullvector of $\hat{\beta}$ and, hence, of $\operatorname{Im}[\hat{\epsilon}]$, and since we assume that $\operatorname{Im}[\hat{\epsilon}]$ has no null vectors, the result is

$$
\operatorname{Im}[\lambda]=\frac{\left\|\beta \mathbf{v}_{\lambda}\right\|^{2}}{\left\|\mathbf{v}_{\lambda}\right\|^{2}}>0
$$

which is $\left(c^{\prime}\right)$.
[1] D. Mugnai, A. Ranfagni, and R. Ruggeri, Phys. Rev. Lett. 84, 4830 (2000).

[2] L. J. Wang, A. Kuzmich, and A. Dogariu, Nature (London) 406, 277-279 (2000).

[3] J. Peatross, S. Glasgow, and M. Ware (unpublished).

[4] L. Brillouin, Wave Propagation and Group Velocity (Academic Press, New York, 1960).

[5] J. Peatross, S. A. Glasgow, and M. Ware, Phys. Rev. Lett. 84, 2370 (2000).

[6] C. G. B. Garrett and D. E. McCumber, Phys. Rev. A 1, 305
(1970).

[7] E. L. Bolda, J. C. Garrison, and R. Y. Chiao, Phys. Rev. A 49, 2938 (1994).

[8] S. Chu and S. Wong, Phys. Rev. Lett. 48, 738 (1982).

[9] S. A. Glasgow and J. B. Peatross (unpublished).

[10] J. D. Jackson, Classical Electrodynamics, 2nd. (Wiley, New York, 1975), pp. 236 and 307.

[11] M. P. van Albada, B. A. van Tiggelen, A. Lagendijk, and A. Tip, Phys. Rev. Lett. 66, 3132 (1991).

[12] G. L. J. A. Rikken and B. A. van Tiggelen, Phys. Rev. Lett. 78, 
847 (1997).

[13] L. D. Landau and E. M. Lifshitz, Electrodynamics of Continuous Media, 2nd ed. (Pergamon, New York, 1984), pp. 274332.

[14] D. R. Smith, W. J. Padilla, D. C. Vier, S. C. Nemat-Nasser, and S. Schultz, Phys. Rev. Lett. 84, 4184 (2000).

[15] J. B. Pendry, A. J. Holden, D. J. Robbins, and W. J. Stewart,
IEEE Trans. Microwave Theory Tech. 47, 2075 (1999).

[16] L. C. Evans, in Partial Differential Equations, Berkely Mathematics, Lecture Notes (Center for Pure and Applied Mathematics, Berkeley, CA, 1994), Vol. 3A, pp. 78-80.

[17] Ann. Phys. (Leipzig) 7 (1998).

[18] G. B. Whitham, Linear and Nonlinear Waves (Wiley, New York, 1974), pp. 113-130. 